\title{
An Uncommon Presentation of a Common Disease: A Review of Gastric Metastasis From Breast Carcinoma
}

\author{
Luis Geada ${ }^{1,2}$, Micaella Kantor ${ }^{3}$, Karthik Mohan ${ }^{4}$, Daniel Weingrad ${ }^{5}$, Luis S. Nasiff ${ }^{3}$ \\ 1. Department of General Surgery, Aventura Hospital and Medical Center, Miami, USA 2. Department of General \\ Surgery, Kendall Regional Medical Center, Miami, USA 3. Department of Gastroenterology, Palm Springs Hospital, \\ Miami, USA 4. Department of Gastroenterology, Palmetto General Hospital, Miami, USA 5. Department of Surgical \\ Oncology, Aventura Hospital and Medical Center, Miami, USA
}

Corresponding author: Luis Geada, luisgeada13@gmail.com

\begin{abstract}
Breast cancer is the most common cancer in women, and the leading cause of cancer-related deaths worldwide. Despite advances in screening and treatment modalities, distant metastasis still develops. Breast cancer metastasis to the gastrointestinal tract is very rare, therefore, its diagnosis, therapeutic strategies, and prognosis pose a clinical problem for clinicians. We summarize the current knowledge regarding the clinicopathological characteristics and diagnostic strategies for metastatic tumors in the stomach of breast origin.
\end{abstract}

Categories: Pathology, Gastroenterology, Oncology

Keywords: breast cancer management, gastric malignancy, breast cancer metastasis, breast cancer

\section{Introduction And Background}

Breast carcinoma is the most common malignancy in women accounting for $25.1 \%$ of all cancers in women and the leading cause of cancer-related death among females worldwide [1]. Despite early recognition by screening and efficacy of new treatment modalities, many patients eventually develop metastatic disease either by locoregional recurrence or distant metastases. The most common sites of metastases include the skeleton, lungs, brain, and liver [2-5]. Metastatic disease to the gastrointestinal tract is rare and poses a clinical problem in both diagnosis and management. Primary malignancies that most commonly metastasize to the stomach include breast cancer (27.9\%), lung cancer (23.8\%), esophageal carcinoma (19.1\%), renal cell carcinoma (7.6\%), and malignant melanoma (7.0\%) [6-8]. Aside from melanoma, which has an unusual predilection for metastasis to the gastrointestinal tract, the other primary sites generally mirror their incidence in the general population [8-10].

Review began 11/08/2020 Review ended 11/30/2020 Published 12/05/2020

\section{๑) Copyright 2020}

Geada et al. This is an open access article distributed under the terms of the Creative Commons Attribution License CC-BY 4.0., which permits unrestricted use, distribution, and reproduction in any medium, provided the original author and source are credited.
Based on clinical and autopsy findings, the reported incidence of gastric metastasis is $0.2 \%-0.7 \%[6,7,11]$. The postmortem frequency of gastric metastasis from breast carcinoma is estimated to be at $0.8 \%-18 \%$. The majority of cases originate from invasive lobular carcinomas (ILCs) [2,12-15].

Only a few cases of metastasis from breast cancer to the gastrum have been noted because of its low incidence. There is little documented on the characteristics, outcomes, and endoscopic findings on metastasis of ILCs to the stomach. The factors involved in prognosis and the particular treatments for these patients needs further investigation because of this [8].

\section{Review}

The gastrointestinal tract is a very uncommon site for breast cancer metastasis. While invasive ductal carcinoma (IDC) represents about $80 \%$ of all breast carcinomas, the majority of gastric metastasis arise from ILCs (65.4\%) and only $24.4 \%$ from IDC [16-22]. In the majority of the cases, the diagnosis of breast cancer precedes the signs and symptoms of metastatic disease $[1,23]$. It has been estimated that the mean time from the diagnosis of breast cancer to reported metastasis is six to seven years. However, it has been reported as long as 20 to 30 years later, even after surgical resection [4,14,24,25]. Disseminated disease is seen in $90 \%-$ $94 \%$ of patients at the time of diagnosis of gastric metastasis. Concomitant metastatic sites commonly include bone (60\%), followed by liver (20\%) and lung (18\%) [12,26-28]. After the stomach, other intraabdominal organs involved, in the order of increasing rarity, include the colon, rectum, and small bowel [17]. Gastric metastasis may also present as primary or peritoneal carcinomatosis, involving the peritoneum, adrenals, and lung pleura [17,23]. There are only a handful of case reports in which gastric metastasis was diagnosed prior to or at the same time as breast carcinoma.

Patients present with a landscape of nonspecific symptoms such as anorexia, dysphagia, early satiety, postprandial bloating, epigastric pain, melena, nausea, and vomiting [12,24,29]. Aside from symptoms being nonspecific, they also mimic the side effects of chemotherapy or other medication, liver metastasis, and 
even hypercalcemia of malignancy [2]. This can further cause a delay in diagnosis or misdiagnosis of the disease. Clinically and endoscopically, it is almost impossible to differentiate primary GI tumors or nonHodgkin lymphoma from metastatic disease [12,29].

The endoscopic features can range from benign-appearing lesions (such as gastritis) to diffuse or ulcerated tumors (mimicking primary gastric carcinoma or lymphoma) [12,27,30]. Currently, there are three main categories of lesions described: localized tumor deposition (18\%), diffuse infiltration (i.e. linitis plastica type or gastritis) (57\%-73\%) and external compression (25\%) $[2,12,15,27]$. The most common presentation is the diffuse type- linitis plastica $[15,27,30]$. This lobular breast cancer-induced syndrome was first described by Cormier et al. in 1980 [26]. It resembles a Borrmann Type 4 advanced gastric cancer with diffuse hypertrophy and sclerosis of the gastric mucosal folds and deep invasion of the submucosal and muscularis propria layers [30-34]. Localized infiltration presents macroscopically with either large ulcers, nodules, or polypoid mass lesions [17,31]. Though infrequent, polypoid lesions may grow large and resemble gastrointestinal stromal tumors $[23,33]$. These localized lesions are often described as "bull's eye" or "target" lesions because the submucosal tumors (SMT) tend to have a central depression covered by an intact mucosa or a raised SMT with an ulcerated center [6,30,34-36]. Interestingly, localized-type nodular lesions are more commonly seen in metastatic ductal-type breast carcinoma [21,28]. External compression can occur when a ring-like tumor forms around the cardia or pylorus, for instance. This may lead to pseudoachalasia or gastric outlet obstruction $[6,35,37,38]$. Very rarely, metastatic infiltration of the duodenum or distal bile ducts has been reported which may present with jaundice $[23,33]$.

Because external metastatic invasion of the stomach often spares the superficial mucosal layer, endoscopic biopsies have an increased false-negative rate $[17,34,39]$. In gastric metastasis secondary to ILC, initial superficial biopsies were reported as normal in up to $46 \%-50 \%$ of the cases because the invasion was limited to the submucosa and seromuscular layers $[23,24]$. If there is a high index of suspicion, non-conventional techniques such as macro-biopsies or endoscopic ultrasound-guided fine-needle aspiration cytology should be used whenever possible $[14,40]$. Morphologically, the infiltrated gastric tissue commonly shows poorly cohesive, round tumor cells with an occasional intracytoplasmic lumen arranged in linear cords between the normal gastric glands [17,41]. These cells resemble signet-ring cell tumors in the WHO classification. It has been suggested that a well-defined univacuolated cytoplasm is relatively specific for ILC metastasis [15,42]. Whereas, multivacuolated cytoplasm suggests a primary gastric signet ring cell carcinoma. However, some data is still equivocal, requiring further investigation $[15,42]$. Commonly, biopsy specimens are also misinterpreted as poorly differentiated gastric carcinoma [15]. Other conditions that mimic signet-ring carcinoma include lymphomas, macrophages, xanthomas, or even gastritis [15,42]. Keratin stains are therefore required to diagnose the presence of invasive tumor cells [17].

In order to obtain the definitive diagnosis of metastatic gastric cancer, immunoprofiling of the tumor cells is imperative. Specific biological immunohistochemical markers for breast, as well as other organs, can aid in the differential diagnosis of neoplasms. Among these, estrogen receptor protein (ER) is the most influential and well-known marker for differentiating metastatic breast cancer. ER is expressed in $72 \%-90 \%$ of breast tumor cells. Progesterone receptor protein (PR) is seen in approximately $33 \%$ of cases $[27,30,34,42]$. However, testing for ER and PR biomarkers alone is not suitable because not all breast cancer cells express these hormone receptors. It has also been widely reported that the receptor expression of the primary breast cancer may be lost in metastatic tissue due to disease progression or following prolonged hormonal therapy [43-45]. In addition, various studies have shown ER and/or PR positivity within primary gastric cells in up to $28 \%$ and $12 \%$ of the cases, respectively [34,46-48]. Velthuysen et al. [44] demonstrated that secondgeneration antibodies against ER-alpha can be more specific and reliable for determining breast origin, as they are not expressed by gastric tumor cells. However, ER-alpha is limited in those with ER-negative breast cancer. The human epidermal growth factor receptor 2 (HER-2) is dysregulated in numerous types of solid tumors including those of breast and gastric origin $[43,45]$. Therefore, its role in diagnosis is also limited and is useful only when tailoring treatment [8].

Other breast specific markers include gross cystic disease fluid protein (GCDFP-15), mammaglobin, and GATA protein type 3 [49]. GCDFP-15 is a pathological secretin released by the breast particularly in the setting of apocrine metaplasia of the breast [49]. It is highly specific for mammary differentiation and yields a specificity of $93 \%-100 \%$ with a sensitivity of $11 \%-76 \%$. It has the highest expression in the lobular carcinoma subtype $[24,30,45]$. Mammaglobin, a mammary gland-specific gene that is overexpressed in breast cancer, has an expression rate of $47.8 \%$ to $80 \%$ in primary and metastatic breast carcinomas [34]. GATA protein type 3 is a multifunctional transcription factor and part of the GATA family of zinc fingers DNA binding proteins. It is only present in breast and urothelial carcinomas [30]. It is expressed in up to 96.6\% of ER-positive breast carcinomas, however, its expression decreases to $21.6 \%$ in triple-negative cases $[15,24]$.

Cytokeratin stains, specifically CK5/6, CK 7, and CK 20, have also been used to aid in differentiating metastatic gastric tumors [15]. CK7+/CK20- are usually expressed in breast, ovarian, and lung adenocarcinomas, however, it can be seen in up to 33\% of gastric adenocarcinoma cases [15,24]. CK7-/CK20+ and CK20+/ER- are patterns typically seen in primary gastric carcinomas [15,24]. CK7 alone has no discriminatory value because it is present in $71 \%$ of gastrointestinal malignancies and $95 \%$ in lobular carcinomas [5]. Positive CK5/6 is present in $61 \%$ of breast cancers and in $16 \%$ of gastric cancers [14]. In a 
large retrospective study that evaluated a broad panel of immunohistochemical stains, O'Connell et al. [45] found CK20+, MUC6, DAS-1 (a monoclonal antibody), and CDX2 (a caudal type homeobox transcription factor) to be $100 \%$ specific for primary gastric carcinomas.

The loss of E-cadherin expression occurs in both invasive lobular carcinoma of the breast and in diffuse-type or poorly cohesive gastric carcinoma [34,44]. Mutations in the $\mathrm{CDH1}$ gene leads to E-cadherin inactivation and subsequently may lead to a familial cancer disorder called hereditary diffuse gastric cancer (HDGC) (12). Patients with HDGC have a 56\%-70\% lifetime risk of developing gastric cancer, and in women, a $42 \%$ lifetime risk of developing lobular carcinoma [12]. Mutations in E-cadherin lead to impaired tumor suppression and cell adhesion which increases the likelihood of tumor cell invasion and metastases. It has been reported that $85 \%$ of ILCs and $50 \%$ of gastric carcinomas have decreased E-cadherin immunoreactivity [44]. Therefore, in the absence of E-cadherin expression, one must differentiate between the presence of two synchronous neoplasia or primary neoplasia with metastases. Even though the exact mechanism regarding the different metastatic patterns between ILCs and IDCs remains obscure, the loss of E-cadherin expression may play a role in the more diffuse metastatic growth pattern of ILCs.

Comparison of endoscopic biopsies with the prior or with the present breast carcinoma specimen improves diagnosis. However, antigen expression may differ between primary and metastatic tumor sites $[2,6,45]$. In numerous studies, patients were diagnosed with metastatic breast carcinomas only after a gastrectomy was performed for primary gastric carcinoma [15,42]. Taken together, relying solely on tumor biomarkers for accurate diagnosis may be vexing given the lack of a specific immunohistochemistry biomarker for breast cancer plus the instability of the tumor phenotype along the disease progression.

It is crucial to distinguish metastatic gastric tumor from primary gastric carcinoma because treatment and prognosis greatly differ between the two. Only a small number of studies have investigated the prognostic factors and standard treatment strategies for gastric metastasis from breast carcinoma because of its low incidence. The presence of gastric metastases reflects advanced disease which makes prognosis poor. Following the diagnosis of gastric metastasis, the median survival rate is estimated to be between 10-28 months $[17,27,19,42]$. Survival is further decreased in those with multiorgan metastasis.

In patients with primary gastric cancer, without distant or peritoneal metastasis, surgical resection is the most effective treatment [34]. Because gastric metastasis from breast cancer is a systemic disease, metastatic gastric cancer is treated systemically with chemotherapy, hormone therapy, or combined therapy. Therefore, hormone receptor status is crucial. The treatment plan is often individualized and based on age, clinical condition, hormone receptor status, and previous treatment [2]. Patients who present with linitis plastic type metastasis and are hormone receptor positive have been shown to respond well to conventional chemotherapy and hormone therapy $[19,27]$. In two large retrospective studies, remission rates were achieved in $46 \%$ to $52 \%$ of the time. This prolonged survival time by two to three years [2,7]. Zelek et al. [50] reported a median survival of 21 months in patients who received chemotherapy. They compared this to less than 12 months in those who underwent surgical gastric resection. Surgical intervention did not significantly improve survival ( 28 vs. 26 months). However, in patients with exclusive gastric metastasis that underwent palliative surgical resection, median survival was prolonged (44 vs 9 months). Unfortunately, this was not statistically significant. Surgical intervention is reserved for patients with unique localized gastric metastasis and palliatively in those with complications such as intestinal obstruction or bleeding $[30,34,50]$. Minimally invasive interventions, such as placement of endoscopic self-expandable metallic stents (SEMS) are being further investigated and used for symptomatic management, for instance, in gastric outlet obstruction caused by metastatic gastric tumor [34].

\section{Conclusions}

To this day, the literature published on metastatic tumors of the stomach is still incomplete, the majority being case reports and few small retrospective studies. Breast cancer metastasis to the gastrointestinal tract, particularly the stomach, can present with a wide range of clinical and radiological findings, often mimicking other more common disorders. Due to the high incidence of breast carcinoma, clinicians should be aware of the possibility of gastric metastasis in an adequate clinical setting. Prompting early investigation can prevent disease progression and complications. Diagnosis poses many challenges, particularly in cases such as ours, where symptoms of gastric metastasis precede the diagnosis of breast carcinoma. Large prospective studies are needed to improve the understanding of the biological and pathological characteristics of these tumors since clinical presentation is unspecific and endoscopic appearance is heterogeneous. The mechanism underlying the metastatic pattern of ILCs is still unclear, however, the loss of expression of E-cadherin, the cell-cell adhesion molecule, in this subtype may contribute to its particular pattern of spread. We believe understanding the specific biological and molecular mechanisms of ILCs will contribute to advances in the development of more specific therapeutic approaches, thus decreasing the rate of metastasis spread. Therefore, there is an urgent need for further investigation on the biological and clinical behavior of breast carcinomas, particularly ILCs given their more aggressive comportment and metastasis to unusual organs, such as the gastrointestinal tract.

\section{Additional Information}




\section{Disclosures}

Conflicts of interest: In compliance with the ICMJE uniform disclosure form, all authors declare the following: Payment/services info: All authors have declared that no financial support was received from any organization for the submitted work. Financial relationships: All authors have declared that they have no financial relationships at present or within the previous three years with any organizations that might have an interest in the submitted work. Other relationships: All authors have declared that there are no other relationships or activities that could appear to have influenced the submitted work.

\section{References}

1. Ghoncheh M, Pournamdar Z, Salehiniya H: Incidence and mortality and epidemiology of breast cancer in the world. Asian Pac J Cancer Prev. 2016, 17:43-46. 10.7314/APJCP.2016.17.S3.43

2. Taal BG, Peterse H, Boot H: Clinical presentation, endoscopic features, and treatment of gastric metastases from breast carcinoma. Cancer. 2000, 89:2214-21. 10.1002/1097-0142(20001201)89:11<2214::AIDCNCR9>3.0.CO;2-D

3. DeVita VT, Lawrence TS, Rosenberg SA: Cancer of the breast Principles \& Practice of Oncology, 10th edition. DeVita VT, Lawrence TS, Rosenberg SA (ed): Lippincott Williams \& Wilkins, Philadelphia; 2015.

4. Nazareno J, Taves D, Preiksaitis HG: Metastatic breast cancer to the gastrointestinal tract: a case series and review of the literature. World J Gastroenterol. 2006, 12:6219-6224. 10.3748/wig.v12.i38.6219

5. Gifaldi AS, Petros JG, Wolfe GRZ: Metastatic breast carcinoma presenting as persistent diarrhea . J Surg Oncol. 1992, 51:211-215. 10.1002/jso.2930510317

6. Namikawa T, Hanazaki K: Clinicopathological features and treatment outcomes of metastatic tumors in the stomach. Surg Today. 2014, 44:1392-9. 10.1007/s00595-013-0671-9

7. Kobayashi O, Murakami H, Yoshida T, et al.: Clinical diagnosis of metastatic gastric tumors: clinicopathologic findings and prognosis of nine patients in a single cancer center. World J Surg. 2004, 28:548-51. 10.1007/s00268-004-7216-8

8. Xu L, Liang S, Yan N, et al.: Metastatic gastric cancer from breast carcinoma: a report of 78 cases . Oncol Lett. 2017, 14:4069-4077. 10.3892/ol.2017.6703

9. Menuck LS, Amberg JR: Metastatic disease involving the stomach . Am J Dig Dis. 1975, 20:903-13. 10.1007/BF01070875

10. Takeuchi H, Hiroshige S, Yoshikawa Y, Kusumoto T, Muto Y: A case of synchronous metastasis of breast cancer to stomach and colon. Anticancer Res. 2012, 32:4051-4055.

11. De Palma GD, Masone S, Rega M, et al.: Metastatic tumors to the stomach: clinical and endoscopic features . World J Gastroenterol. 2006, 12:7326-8. 10.3748/wjg.v12.i45.7326

12. El-Hage A, Ruel C, Afif W, et al.: Metastatic pattern of invasive lobular carcinoma of the breast-emphasis on gastric metastases. J Surg Oncol. 2016, 114:543-547. 10.1002/jso.24362

13. Veronesi U, Goldhirsch A, Veronesi P, Gentilini OD, Leonardi MC: Breast Cancer: Innovations in Research and Management. Veronesi P, Leonardi M, Gentilini O (ed): Springer, Cham, Switzerland; 2017. 10.1007/9783-319-48848-6

14. Schwarz RE, Klimstra DS, Turnbull AD: Metastatic breast cancer masquerading as gastrointestinal primary . Am J Gastroenterol. 1998, 93:111-114. 10.1111/j.1572-0241.1998.111_c.X

15. Gurzu S, Banias L, Bara T, Feher I, Bara T Jr, Jung I: The epithelial-mesenchymal transition pathway in two cases with gastric metastasis originating from breast carcinoma, one with a metachronous primary gastric cancer. Recent Pat Anticancer Drug Discov. 2018, 13:118. 10.2174/2212798409666171101121108

16. Van Trappen P, Serreyn R, Elewaut AE, Cocquyt V, Van Belle S: Abdominal pain with anorexia in patients with breast carcinoma. Ann Oncol. 1998, 9:1243-1245. 10.1023/a:1008287007819

17. Almubarak MM, Lae M, Cacheux W, et al.: Gastric metastasis of breast cancer: a single centre retrospective study. Dig Liver Dis. 2011, 43:823-827. 10.1016/j.dld.2011.04.009

18. Shimizu S, Matsukawa H, Iwasaki H, Fukagawa H, Masukawa K, Horiguchi K, Ohaki Y: A case of gastric metastases from carcinoma of the breast [Article in Japanese]. Gan No Rinsho. 1988, 34:1163-8.

19. McLemore EC, Pockaj BA, Reynolds C, Gray RJ, Hernandez JL, Grant CS, Donohue JH: Breast cancer: presentation and intervention in women with gastrointestinal metastasis and carcinomatosis. Ann Surg Oncol. 2005, 12:886-94. 10.1245/ASO.2005.03.030

20. Borst MJ, Ingold JA: Metastatic patterns of invasive lobular versus invasive ductal carcinoma of the breast . Surgery. 1993, 114:641-2.

21. Harris M, Howell A, Chrissohou M, Swindell RI, Hudson M, Sellwood RA: A comparison of the metastatic pattern of infiltrating lobular carcinoma and infiltrating duct carcinoma of the breast. Br J Cancer. 1984, 50:23-30. 10.1038/bjc.1984.135

22. Taal BG, den Hartog Jager FC, Steinmetz R, Peterse H: The spectrum of gastrointestinal metastases of breast carcinoma: I. Stomach. Gastrointest Endosc. 1993, 39:130-35. 10.1016/s0016-5107(92)70377-0

23. Hussain T, Elahi B, McManus P, Mahapatra T, Kneeshaw PJ: Gastric obstruction secondary to metastatic breast cancer: a case report and literature review. J Med Case Rep. 2012, 6:232. 10.1186/1752-1947-6-232

24. Kim DH, Son SM, Choi YJ: Gastric metastasis from invasive lobular breast cancer, mimicking primary gastric cancer: a case report. Medicine. 2018, 97:e0258. 10.1097/MD.0000000000010258

25. Libânio D, Dinis-Ribeiro M, Pimentel-Nunes P: Gastric metastasis of breast cancer after 20 years . GE Port J Gastroenterol. 2018, 25:99-101. 10.1159/000479233

26. Cormier WJ, Gaffey TA, Welch JM, Welch JS, Edmonson JH: Linitis plastica caused by metastatic lobular carcinoma of the breast. Mayo Clin Proc. 1980, 55:747-53.

27. Taal BG, Westerman H, Boot H, Rankin EM: Clinical and endoscopic features of melanoma metastases in the upper GI tract. Gastrointest Endosc. 1999, 50:261-263. 10.1016/s0016-5107(99)70236-1

28. Jones GE, Strauss DC, Forshaw MJ, Deere H, Mahedeva U, Mason RC: Breast cancer metastasis to the stomach may mimic primary gastric cancer: report of two cases and review of literature. World J Surg Oncol. 2007, 5:75. 10.1186/1477-7819-5-75 
29. Gurzu S, Beleaua MA, Banias L, Jung I: Gastric metastases mimicking primary gastric cancer: a brief literature review. Cancer Transl Med. 2017, 3:101-105. 10.4103/ctm.ctm_67_16

30. Ricciuti B, Leonardi GC, Ravaioli N, et al.: Ductal breast carcinoma metastatic to the stomach resembling primary linitis plastica in a male patient. J Breast Cancer. 2016, 19:324-329. 10.4048/jbc.2016.19.3.324

31. Taal BG, Boot H, van Heerde P, de Jong D, Hart AA, Burgers JM: Primary non-Hodgkin lymphoma of the stomach: endoscopic pattern and prognosis in low versus high grade malignancy in relation to the MALT concept. Gut. 1996, 39:556-61. 10.1136/gut.39.4.556

32. Oda I, Kondo H, Yamao T, et al.: Metastatic tumors to the stomach: analysis of 54 patients diagnosed at endoscopy and 347 autopsy cases. Endoscopy. 2001, 33:507-510. 10.1055/s-2001-14960

33. Taal BG, den Hartog Jager FCA, Steinmetz R, Peterse H: The spectrum of gastrointestinal metastases of breast carcinoma: II. The colon and rectum. Gastrointest Endosc. 1992, 38:136-141. 10.1016/s00165107(92)70378-2

34. Yagi Y, Sasaki S, Yoshikawa A, et al.: Metastatic gastric carcinoma from breast cancer mimicking primary linitis plastica: a case report. Oncol Lett. 2015, 10:3483-3487. 10.3892/ol.2015.3788

35. Bankar S, Patkar S, Desai S, Shrikhandes SV: Unusual presentation of melanoma of unknown primary origin: a case report and review of literature. J Cancer Res Ther. 2015, 11:1025. 10.4103/0973-1482.148680

36. Yamashita K, Takeno S, Nimura S, et al.: Gastric metastasis from salivary duct carcinoma mimicking primary gastric cancer. Int J Surg Case Rep. 2016, 23:36-9. 10.1016/j.ijscr.2016.04.004

37. Daniels IR, Layer GT, Chisholm EM: Lessons to be learned: a case study approach: bowel obstruction due to extrinsic compression by metastatic lobular carcinoma of the breast. J R Soc Promot Health. 2002, 122:61-2. $10.1177 / 146642400212200118$

38. Reeder MM, Cavanagh RC: “Bull's eye” lesions: solitary or multiple nodules in the gastrointestinal tract with large central ulceration. JAMA. 1974, 229:825-826. 10.1001/jama.1974.03230450057032

39. Pectasides D, Psyrri A, Pliarchopoulou K, et al.: Gastric metastases originating from breast cancer: report of 8 cases and review of the literature. Anticancer Res. 2009, 29:4759-4763.

40. Ulmer LL, Cormier I, Jha LK, Singh S, Fisher KW, Hewlett AT: Use of endoscopic ultrasound in a diagnostic dilemma: metastatic breast cancer to the stomach. Case Rep Gastroenterol. 2018, 3: 10.1155/2018/2820352

41. Fenoglio-Preiser C, Carneiro F, Correa P, et al.: Gastric carcinoma. Pathology \& genetics: tumors of the digestive system. World Health Organization classification of tumours. Pathology and genetics of tumours of the digestive system. Hamilton SR, Aaltonen LA (ed): IARC Press, France; 2000. 39-52. 10.1.1.832.1047

42. Ciulla A, Castronovo G, Tomasello G, Maiorana AM, Russo L, Daniele E, Genova G: Gastric metastases originating from occult breast lobular carcinoma: diagnostic and therapeutic problems. World J Surg Oncol. 2008, 6:78. 10.1186/1477-7819-6-78

43. Szekely B, Nagy ZI, Farago Z, et al.: Comparison of immunophenotypes of primary breast carcinomas and multiple corresponding distant metastases: an autopsy study of 25 patients. Clin Exp Metastasis. 2017, 34:103-13. 10.1007/s10585-016-9830-x

44. van Velthuysen ML, Taal BG, van der Hoeven JJ, Peterse JL: Expression of oestrogen receptor and loss of Ecadherin are diagnostic for gastric metastasis of breast carcinoma. Histopathology. 2005, 46:153-7. 10.1111/j.1365-2559.2005.02062.x

45. O’Connell FP, Wang HH, Odze RD: Utility of immunohistochemistry in distinguishing primary adenocarcinomas from metastatic breast carcinomas in the gastrointestinal tract. Arch Pathol Lab Med. 2005, 129:338-47.

46. Kojima O, Takahashi T, Kawakami S, Uehara Y, Matsui M: Localization of estrogen receptors in gastric cancer using immunohistochemical staining of monoclonal antibody. Cancer. 1991, 67:2401-2406. 10.1002/1097-0142(19910501)67:9<2401::aid-cncr2820670931>3.0.c0;2-h

47. Wu CW, Tsay SH, Chang TJ, et al.: Clinicopathologic comparisons between estrogen receptor-positive and negative gastric cancers. J Surg Oncol. 1992, 51:231-235. 10.1002/jso.2930510406

48. Harrison JD, Jones JA, Ellis IO, et al.: Oestrogen receptor D5 antibody is an independent negative prognostic factor in gastric cancer. Br J Surg. 1991, 78:334-336. 10.1002/bjs.1800780321

49. Mazoujian G, Pinkus GS, Davis S, Haagensen DE Jr: Immunohistochemistry of a gross cystic disease fluid protein (GCDFP-15) of the breast. A marker of apocrine epithelium and breast carcinomas with apocrine features. Am J Pathol. 1983, 110:105-112.

50. Zelek L, Cottu PH, Mignot L, et al.: Gastric metastases from breast cancer: A retrospective series of 12 patients. Am J Clin Oncol. 2001, 24:363-5. 10.1097/00000421-200108000-00009 